Case Report:

\title{
Carpal Tunnel Syndrome Associated with Klippel-Trénaunay Syndrome: A Case Report
}

\author{
Joan Arenas-Prat ${ }^{1^{*}}$ (D)
}

1. Serveis Mèdics Penedès, Department of Orthopedics, Barcelona, Catalonia, Spain

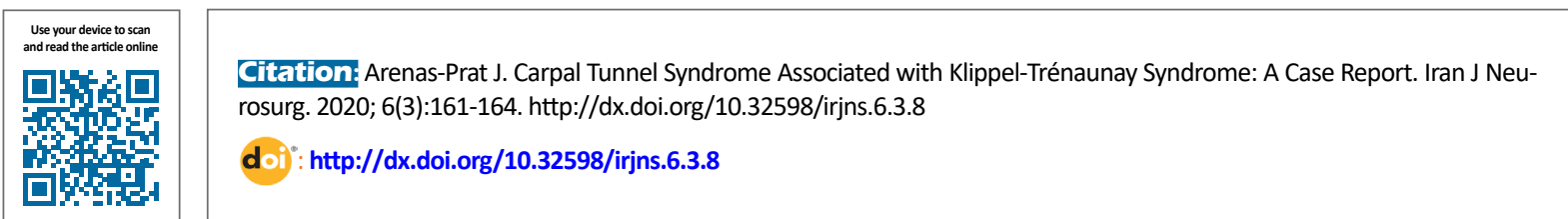

\section{(c) (1)}

Article info:

Received: 05 Jan 2020

Accepted: 25 Apr 2020

Available Online: $01 \mathrm{Jul} 2020$

Keywords:

Klippel-Trénaunay syndrome,

Carpal tunnel syndrome,

Median nerve

\section{ABSTRACT}

Background and Importance: Klippel-Trénaunay syndrome is a rare congenital condition that rarely affects peripheral nerves. Median nerve involvement at the carpal tunnel level has only been reported on four occasions of this syndrome in the medical literature.

Case Presentation: A 61-year-old Caucasian female patient with Klippel-Trénaunay syndrome presented with a 10-month history of paraesthesia and numbness affecting the median nerve distribution area of her left hand. The clinical and neurophysiological examination confirmed a moderately severe carpal tunnel syndrome that required surgical decompression.

Conclusion: Pre-operative findings demonstrated diffuse vascular infiltration and engorgement of the median nerve. Flexor tendons had a normal appearance. The patient had a satisfactory post-operative period with full resolution of her symptoms 4 weeks after the procedure. Although peripheral nerves are rarely affected in patients with Klippel-Trénaunay syndrome, neurological symptoms could indicate nerve involvement and Magnetic Resonance Imaging (MRI) examination should be considered to further assess the extension of the lesion.

\section{${ }^{*}$ Corresponding Author:}

Joan Arenas-Prat, MD.

Address: Serveis Mèdics Penedès, Department of Orthopedics, Barcelona, Catalonia, Spain

Tel: +98 (938) 172299

E-mail: arenasprat@hotmail.com 


\section{Highlights}

- This study focuses on peripheral nerve involvement in Klippel-Trénaunay syndrome.

- Carpal tunnel syndrome is associated with Klippel-Trénaunay syndrome.

\section{Plain Language Summary}

This report presents the operative findings of a carpal tunnel decompression in a patient suffering from KlippelTrénaunay syndrome where the median nerve had an abnormal appearance consisting of diffuse vascular infiltration. The medical literature is also reviewed together with the association of nerve abnormalities in patients affected with this rare congenital condition. We feel that patients affected by Klippel-Trénaunay syndrome that complain of abnormal sensation, sonographic, or MRI imaging should be considered to rule out nerve involvement.

\section{Background and Importance}

lippel-Trénaunay syndrome is an uncommon congenital condition that can occasionally affect peripheral nerves [1]. In the medical literature, the median nerve has only been found to be affected in 4 cases [2-5], but despite this, we believe that sonographic or MRI imaging of the carpal tunnel and the median nerve should be considered pre-operatively.

\section{Case presentation}

We present the case of a 61-year-old patient with Klippel-Trénaunay syndrome complaining of a 10-month history of paresthesia affecting the median nerve distribution of her left hand. The clinical and neurophysiological examination confirmed the diagnosis of carpal tunnel syndrome and the patient was operated on, with full resolution of her symptoms.

Klippel-Trénaunay syndrome is an uncommon congenital condition that causes hypertrophy of bone and soft tissue, varicose veins, and cutaneous capillary malformation developing cutaneous port-wine colored stains. Carpal tunnel syndrome associated with this rare condition has only been described in 4 cases in the medical literature.

A 61-year-old female patient with Klippel-Trénaunay syndrome presented to our department with a 10-month history of paresthesia affecting the median nerve distribution of her left hand. Her symptoms got worse gradually interfering with her daily activities and sleep. She had no further relevant past medical history.
On examination, the patient presented the classical port-wine stains distributed on the entire left upper extremity skin. At hand level, she had a normal abductor pollicis brevis muscle with positive Tinel, Durkan, and Phalen tests. Neurophysiological investigations confirmed a moderate reduction of the median nerve conduction velocities across the left wrist.

The patient was taken to the theater for carpal tunnel release under local anesthesia. Inspection of the median nerve revealed a diffuse vascular infiltration and ingurgitation with an enlarged diameter of the nerve and indentation with hourglass deformity (Figure 1). Flexor tendons had a normal appearance. The immediate post-operative period was uneventful and two weeks later, the patient was reviewed for suture removal and gradual mobilization. At that time, most neurological symptoms had already improved and the patient was discharged 4 weeks after the operation with full resolution of symptoms with an open appointment.

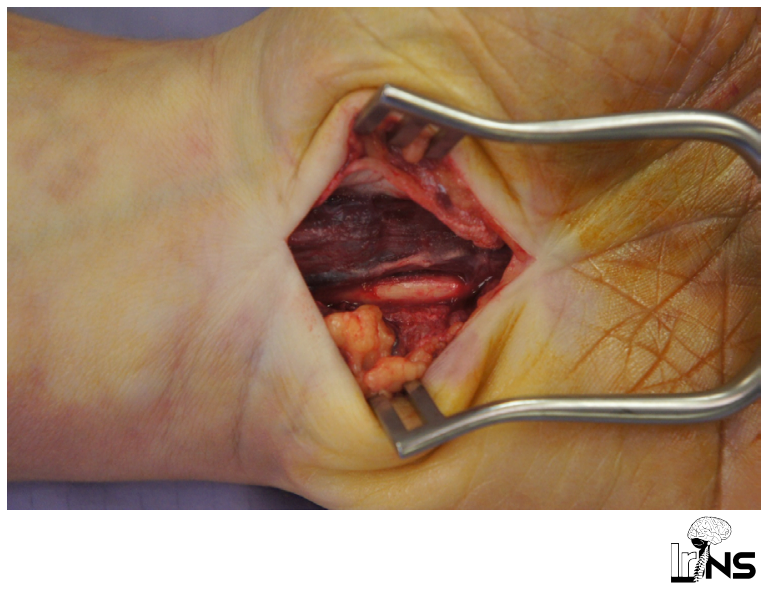

Figure 1. Median nerve showing diffuse vascular infiltration and ingurgitation with enlarged diameter and indentation with hourglass deformity 
Table 1. Documented cases of median nerve compression at the carpal tunnel level, associated with Klippel-Trénaunay syndrome

\begin{tabular}{crr}
\hline Authors & Journal and Year of Publication & Cause of Median Nerve Compression \\
\hline Matsubara et al. [2] & Journal of Bone and Joint Surgery, Am; 2009 & Fibrolipomatous Hamartoma \\
Awada et al. [3] & Revue Neurologie (Paris); 2003 & Lymphatic abnormal proliferation \\
Gassmann and Mettler [4] & Handchirurgie Mikrochirurgie Plastische Chirurgie; 2001 & Cavernous haemangioma \\
Siegall and Ratner [5] & Journal of Bone and Joint Surgery, Am; 2015 & No relevant macroscopic findings
\end{tabular}

\section{Discussion}

Klippel-Trénaunay syndrome is a rare congenital condition characterized by hypertrophy of bone and soft tissue, varicose veins, and cutaneous capillary malformation developing the classical skin port-wine stains [1]. Carpal tunnel syndrome is seldom associated with Klippel-Trénaunay syndrome and only 4 cases (Table 1) have been reported in the medical literature [2-5]. This is explained by the fact that peripheral neuropathy is rare in Klippel-Trénaunay syndrome. It is related to the presence of epineural microscopic arteriovenous anastomoses and endoneurial vascular coils which corresponds to the macroscopic findings of the case presented in this article.

Lymphatic obstruction, fibrolipomatous hamartoma, and cavernous hemangioma have been described as pathogenesis in this type of neuropathy. Furthermore, the risk of malignancy of these vascular nerve lesions has been described [6].

Despite its rare association with peripheral neuropathy, we believe that patients with Klippel-Trénaunay syndrome who complain of persistent paresthesia in a specific nerve distribution would benefit from a further diagnostic tool such as MRI to fully assess the extent of the disease. A Tinel test would be relevant to determine the site of imaging of the relevant nerve.

\section{Conclusion}

Patients with Klippel-Trénaunay syndrome who complain of neurological symptoms, apart from compressive neuropathies, should be assessed for nerve involvement secondary to their congenital condition. Clinical examination including the Tinel test and neurophysiology studies together with imaging techniques suc $h$ as MRI will give the physician and surgeon the full picture of the condition. Nerve infiltration with epineural arte- riovenous anastomoses and endoneurial vascular coils will inevitably cause an enlarged nerve diameter that can lead eventually to nerve compression in sites where this is anatomically feasible.

\section{Ethical Considerations}

\section{Compliance with ethical guidelines}

Pre-operative written informed consent was obtained from the patient to take the images.

Funding

This research did not receive any grant from funding agencies in the public, commercial, or non-profit sectors.

\section{Conflict of interest}

The author declared no conflicts of interest.

\section{Acknowledgements}

I thank Mrs. Marta Bernet, Theatre Nurse, for taking the pictures.

\section{References}

[1] Boyle J. Congenital disease of the vasculature. In: Thomson $\mathrm{M}$, editor. Oxford textbook of vascular surgery. Oxford: Oxford University Press; 2016. https://www.google.com/ books/edition/Oxford_Textbook_of_Vascular_Surgery/0IL ADAAAQBAJ?hl=en\&gbpv $=0$

[2] Matsubara M, Tanikawa H, Mogami Y, Shibata Sh, Uchiyama Sh, Kato H. Carpal tunnel syndrome due to fibrolipomatous hamartoma of the median nerve in Klippel-Trénaunay syndrome. A case report. The Journal of Bone \& Joint Surgery. 2009; 91(5):1223-7. [DOI:10.2106/JBJS.H.00813] [PMID] 
[3] Awada A, Al Jumah M, Al Ayafi H. [Two unusual complications of the Klippel-Trénaunay syndrome: Carpal tunnel syndrome and cerebral venous thrombosis (French)]. Revue Neurologique (Paris). 2003; 159(6-7 Pt 1):688-90. [PMID]

[4] Gassmann NB, Mettler M. [Klippel-Trenaunay syndrome as a rare cause of carpal tunnel syndrome: Case report (German)]. Handchirurgie, Mikrochirurgie, Plastische Chirurgie. 2001; 33(1):49-51. [DOI:10.1055/s-2001-12073] [PMID]

[5] Siegall E, Ratner J. Carpal tunnel syndrome associated with Klippel-Trénaunay-Weber syndrome: A case report. JBJS Case Connector. 2015; 5(2):e47. [DOI:10.2106/JBJS. CC.N.00133] [PMID]

[6] Ploegmakers MJM, Pruszczynski M, De Rooy J, Kusters $\mathrm{B}$, Veth RPH. Angiosarcoma with malignant peripheral nerve sheath tumour developing in a patient with KlippelTrénaunay-Weber syndrome. Sarcoma. 2005; 9(3/4):584623. [DOI:10.1080/13577140500353743] [PMID] [PMCID] 